\title{
OPTICAL DIFFERENCES BETWEEN RADIO-LOUD AND RADIO-QUIET QSOS
}

\author{
PAUL J. FRANCIS \\ Steward Observatory, University of Arizona \\ Tucson AZ 85721, U.S.A.*
}

\begin{abstract}
We analyse the rest-frame UV spectra of a complete sample of optically selected radio-loud and radio-quiet QSOs. Our results are:

1: Broad absorption-line QSOs (BALQSOs) are all radio quiet, but they are strongly clustered toward the top end of the radio-quiet population in radio power.

2: Radio-loud QSOs have higher equivalent-width, narrower high ionisation emission-lines than radio-quiet $Q S O s$.
\end{abstract}

\section{Observations}

We observed 250 of the brightest QSOs in the optically selected Large Bright QSO Survey with the MMT and VLA. A clearly bimodal distribution of radio-to-optical flux ratios was found.

\section{Results}

\subsection{Absorption Lines}

None of the radio-loud QSOs showed broad absorption lines (BALs). However, the BAL QSOs were not uniformly distributed through the radio-quiet population; they were strongly clustered towards the highest radio-to-optical flux ratios of the radio-quiet population ( $99.99 \%$ confidence). This result can be explained if, for radio-quiet QSOs, the BAL covering factor is proportional to the radio flux.

\subsection{EMISSION-LINES}

We studied the Lyman- $\alpha$ (1216), C IV (1549) and C III] (1909) emission-lines. In radio-loud QSOs they had $\sim 30 \%$ higher mean equivalent-widths (97\% confidence) and were $30 \%$ narrower (96\% confidence) than in radio-quiet QSOs. This is evidence for more extended emission-line regions in radio-loud QSOs.

\section{Further Reading}

See Francis, Hooper \& Impey, 1993, Astronomical Journal 106, 417 and references therein for more details and discussion.

\footnotetext{
* Current Address: School of Physics, University of Melbourne, Parkville, Victoria, Australia 3052
}

T. J.-L. Courvoisier and A. Blecha: Multi-Wavelength Continuum Emission of AGN, 503.

(C) 1994 IAU. Printed in the Netherlands. 